\title{
Multimorbidity by Patient and Tumor Factors and Time-to-Surgery Among Colorectal Cancer Patients in Spain: A Population-Based Study
}

This article was published in the following Dove Press journal: Clinical Epidemiology

\author{
Miguel Angel Luque-Fernandez ${ }^{1-3}{ }^{1-3}$ \\ Daniel Redondo-Sanchez ${ }^{1,3,4}$ \\ Shing Fung Lee 5 \\ Miguel Rodríguez-Barranco 1,3,4 \\ $M^{\text {a }}$ Carmen Carmona-García (iD) ${ }^{6-8}$ \\ Rafael Marcos-Gragera (iD $3,6,7,9$ \\ María-José Sánchez (iD) 1,3,4 \\ 'Non-Communicable Disease and Cancer \\ Epidemiology Group, Instituto de \\ Investigación Biosanitaria de Granada ibs. \\ GRANADA, University of Granada, \\ Granada, Spain; ${ }^{2}$ Non-Communicable \\ Disease Epidemiology, London School of \\ Hygiene and Tropical Medicine, London, UK; \\ ${ }^{3}$ Biomedical Network Research Centers of \\ Epidemiology and Public Health (CIBERESP), \\ Madrid, Spain; ${ }^{4}$ Andalusian School of Public \\ Health, Granada, Spain; ${ }^{5}$ Department of \\ Clinical Oncology, Tuen Mun Hospital, New \\ Territories West Cluster, Hospital \\ Authority, Hong Kong; ${ }^{6}$ Catalan Institute of \\ Oncology, Epidemiology Unit and Girona \\ Cancer Registry, Oncology Coordination \\ Plan, Department of Health, Autonomous \\ Government of Catalonia, Catalan Institute \\ of Oncology, Girona, Spain; ${ }^{7}$ Descriptive \\ Epidemiology, Genetics and Cancer \\ Prevention Group, Biomedical Research \\ Institute (IDIBGI), Girona, Spain; \\ ${ }^{8}$ Department of Medical Oncology, Institut \\ Català d'Oncologia Hospital Universitari de \\ Girona Dr. Josep Trueta, Girona, Spain; \\ ${ }^{9}$ Research Group on Statistics, \\ Econometrics and Health (GRECS), \\ University of Girona, Girona, Spain
}

Correspondence: Miguel Angel LuqueFernandez

Andalusian School of Public Health, Cuesta Del Observatorio, 4, Granada 18080, Spain

Email miguel-angel.luque@Ishtm.ac.uk
Background: Cancer treatment and outcomes can be influenced by tumor characteristics, patient overall health status, and comorbidities. While previous studies have analyzed the influence of comorbidity on cancer outcomes, limited information is available regarding factors associated with the increased prevalence of comorbidities and multimorbidity among patients with colorectal cancer in Spain.

Patients and Methods: This cross-sectional study obtained data from all colorectal cancer cases diagnosed in two Spanish provinces in 2011 from two population-based cancer registries and electronic health records. We calculated the prevalence of comorbidities according to patient and tumor factors, identified factors associated with an increased prevalence of comorbidity and multimorbidity, analyzed the association between comorbidities and time-to-surgery, and developed an interactive web application (https://comcor.netlify.com/).

Results: The most common comorbidities were diabetes (23.6\%), chronic obstructive pulmonary disease (17.2\%), and congestive heart failure (14.5\%). Among all comorbidities, $52 \%$ of patients were diagnosed at more advanced stages (stage III/IV). Patients with advanced age, restricted performance status or who were disabled, obese, and smokers had a higher prevalence of multimorbidity. Patients with multimorbidity had a longer time-tosurgery than those without comorbidity (17 days, 95\% confidence interval: 3-29 days).

Conclusion: We identified a consistent pattern of factors associated with a higher prevalence of comorbidities and multimorbidity at diagnosis and an increased time-to-surgery among patients with colorectal cancer with multimorbidity in Spain. This pattern may provide insights for further etiological and preventive research and help to identify patients at a higher risk for poorer cancer outcomes and suboptimal treatment.

Keywords: colorectal cancer epidemiology, comorbidity, multimorbidity, elderly

\section{Introduction}

Globally, cancer accounted for 9.6 million deaths in 2018 and was the second most common cause of death. ${ }^{1}$ Colorectal cancer (CRC) is the most frequently diagnosed cancer in Spain. ${ }^{2,3}$ Older CRC patients are underrepresented in clinical trials largely related to their high prevalence of comorbidity. ${ }^{4}$

Comorbidity describes a long-term health condition or disorder occurring alongside the primary disease of interest, such as cancer, ${ }^{5}$ whereas multimorbidity refers to the existence of two or more comorbid conditions. ${ }^{6}$ Comorbidity and multimorbidity are increasingly seen as a problem of the elderly but have also been increasingly reported at a younger age in patients with lower socioeconomic status. ${ }^{7,8}$ 
Despite the common coexistence of comorbidity and cancer, the guidelines and delivery of cancer care generally focus on single disease management. ${ }^{9}{ }^{10}$ However, effective management of comorbid conditions is important in optimizing patients' health status ${ }^{11}$ and decisions regarding cancer treatment require careful consideration of comorbidities. ${ }^{12-14}$ Furthermore, postoperative complications occur more frequently in patients with comorbidity ${ }^{15}$ and certain comorbid conditions have been linked to adverse outcomes following surgery for cancer. ${ }^{12,16}$

While evidence of the influence of comorbidities on cancer outcomes is consistent, little is known about factors associated with a higher prevalence of comorbidities among CRC patients and their association with the time from cancer diagnosis to surgery. Thus, the present study determined the prevalence of individual comorbidities, characterized patient and tumor factors associated with a higher prevalence of individual comorbidities and multimorbidity at diagnosis, and analyzed the association between comorbidities and time-to-surgery among CRC patients at diagnosis in two Spanish provinces (Granada and Girona) in 2011. Furthermore, we studied the extent to which the prevalence of comorbidities among CRC patients was similar to the prevalence in the general Spanish population in 2011.

\section{Materials and Methods}

Study Design, Participants, Data, and

\section{Setting}

This population-based cross-sectional study included all the primary CRC incident cases diagnosed in 2011 in two Spanish population-based cancer registries (Girona and Granada), registered with codes $\mathrm{C} 18-\mathrm{C} 21$ according to the International Classification of Diseases for Oncology, third edition (ICD-O-3). ${ }^{17}$

Comorbidity information and clinical data were obtained retrospectively from a review of patient electronic health records (EHRs) including primary care, pathology, outpatient, and in-patient hospital information. The data collection followed a detailed protocol from the European HighResolution studies collaboration (TRANSCAN-HIGHCARE project within ERA-Net). ${ }^{18}$ Aggregated data to study the prevalence of comorbidities among the general Spanish population by age groups in 2011 were obtained from the Spanish Primary Care Clinical database from the Spanish Ministry of Health, Consumer Affairs and Social Welfare available at https://pestadistico.inteligenciadegestion.mscbs.es/.
The study proposal (CP17/00206) was approved by the internal review board of the Andalusian School of Public Health and the ethics committee from the Department of Health of the Andalusian Regional Government (study 0072-N-18). The study was carried out in accordance with the principles of the Declaration of Helsinki. No samples were used, all data accessed for the study were fully anonymized and the informed consent was waived. The data are held by the Regional Government of Andalusia and the Andalusian Health Department.

\section{Variables}

We recorded patient age, sex, smoking status, body mass index (BMI), performance status, comorbidities, and multimorbidity. Age at diagnosis was categorized into four groups: $<55$, 55-64, 65-74, and $\geq 75$ years. Smoking status was categorized as current, previous, and never smoker. BMI was categorized as underweight-normal $\left(<25.0 \mathrm{~kg} / \mathrm{m}^{2}\right)$, overweight $\left(\geq 25.0 \mathrm{~kg} / \mathrm{m}^{2}\right.$ and $\left.<30 \mathrm{~kg} / \mathrm{m}^{2}\right)$, and obese $\left(\geq 30 \mathrm{~kg} / \mathrm{m}^{2}\right)$. We combined the underweight and normal-weight categories because of data sparsity (fewer than five patients were underweight). The patients' performance status was ascertained based on their medical records using the Eastern Cooperative Oncology Group (ECOG) scale (Supplementary Table S1) ${ }^{20}$

Comorbidities were assessed from patient EHRs using the International Classification of Diseases (ICD)-10 codes (Supplementary Table S2). All comorbidities recorded in the EHRs were included except for those diagnosed within 6 months before cancer diagnosis to prevent the inclusion of CRC-related comorbidities. ${ }^{21}$ The CRC patients' comorbidities were classified based on the Royal College of Surgeons-modified Charlson score, which reduces the number of comorbidities to 12 , removes a category (peptic ulcer disease), and groups diseases (e.g., diabetes mellitus codes with or without complications are grouped into a single category). The score does not assign weights to individual comorbidities. ${ }^{22}$ The final score simply counts the total number of comorbidities for each patient as no comorbidities (0), one comorbidity (1), and two or more $(\geq 2)$ comorbidities, with $(\geq 2)$ comorbidities defined as multimorbidity. ${ }^{22}$

We recorded the tumor topography, morphology, and grade of differentiation, which were coded according to ICD-O-3. The final-stage variable was defined as the combination of clinical and pathological TNM stages and categorized into four groups, based on the seventh edition of the TNM manual (AJCC staging system). ${ }^{23}$ 
The time from cancer diagnosis to surgery (time-tosurgery) was calculated as the number of days elapsed from the date of cancer diagnosis to the date of surgical intervention.

\section{Statistical Analysis}

First, we calculated the prevalence of each comorbidity. However, we only present here the results for 10 comorbidities, as HIV and hemiplegia/paraplegia were only represented by four and three cases, respectively. Then, we calculated the counts and proportions of these comorbidities by patient and tumor factors using chi-square and Fisher's exact tests for statistical inference and the score test of trend for the assessment of linear trends.

To characterize the patient and tumor factors associated with a higher prevalence of multimorbidity $(\geq 2$ chronic conditions vs none) and comorbidity (one chronic condition vs none) at diagnosis, we used a multinomial regression to derive crude and adjusted (for sex and age) prevalence ratios (PRs) with their respective $95 \%$ confidence intervals (CIs) using the category of non-comorbidities as the reference. The PRs indicated the magnitude of the prevalence of comorbidity and multimorbidity among a group of CRC patients with a particular patient or tumor factor relative to another group without the given factor at the moment of cancer diagnosis. ${ }^{24}$ Furthermore, we compared the prevalence of the most common comorbidities among CRC patients with the prevalence of comorbidities among the Spanish general population by age groups.

We used medians and interquartile ranges (IQRs) to describe the time-to-surgery according to patient age, stage, and comorbidity status. Finally, we used a nonparametric robust regression to analyze the association of comorbidities and multimorbidity with time-to-surgery. Nonparametric regression models the mean of the outcome conditional on the covariates but, unlike linear regression, it makes no assumptions about the functional form of the relationship between the outcome and the covariates. ${ }^{25,26}$ We used timeto-surgery in days as the dependent variable and the Royal College of Surgeons-modified Charlson score as the independent variable adjusted for age and cancer stage. We used cross-validation to choose the best data bandwidth for the local linear regression to estimate the time-to-surgery conditional mean as a function of the changes in age and cancer stage. ${ }^{25,26}$ We used 1000 bootstrapped samples to compute the $95 \%$ CIs. Then, we derived the model's predicted marginal time-to-surgery mean and plotted it as a function of age and cancer stage.
We assumed that missing information was completely at random and performed a complete case analysis. We used Stata v.15.1 (StataCorp, College Station, Texas, US) and the commands "npregress", "margins", and "marginsplot" for statistical analysis and developed an interactive web application presenting the results of the study (https://watzilei.com/ $\underline{\text { shiny/CoMCoR/). }}$.

\section{Results}

\section{Patient and Tumor Characteristics}

Diabetes mellitus, chronic obstructive pulmonary disease (COPD), and congestive heart failure were the most common comorbidities among the $1061 \mathrm{CRC}$ cases included in the study $(24 \%, 17 \%$, and $15 \%$, respectively) (Supplementary Table S3).

Supplementary table S4 shows the sociodemographic and tumor characteristic from the $1061 \mathrm{CRC}$ cases. Sixty-one percent of the CRC cases were men and 67\% were aged $>65$ years. Furthermore, $40.4 \%$ of CRC cases were previous or current smokers, $49.0 \%$ were overweight or obese, and $11.8 \%$ had a restricted performance status (i.e., unable to work and limited self-care). The tumor anatomical sites included the right colon (33.6\%), left colon (32.1\%), rectum $(33.3 \%)$, and unspecified (1.0\%). The grades of tumor differentiation were mostly grade two (56.2\%); however, $18.9 \%$ of the tumors were not graded. Only $15.8 \%$ of CRC patients had a stage I tumor at diagnosis and $52.1 \%$ of cases were identified as stage III/IV. More than half (58.9\%) of CRC patients had one or more comorbidities and 30.5\% had multimorbidity. Among patients with multimorbidity, the maximum number of comorbidities was six (in four patients) (Supplementary Table S4). The prevalence of the most common comorbidities among CRC patients was remarkably higher than in the Spanish general population for all the categories of age in 2011. For instance, the prevalence of diabetes among CRC patients $>74$ years was approximately 3 times than in the group of the same age in the overall Spanish population, i.e., $26.4 \%$ vs $8.9 \%$ (Supplementary Table S5).

\section{Patient Factors}

Table 1 shows the distribution and frequency of patient and tumor factors for the top five comorbidities plus dementia. Overall, there was a gradient in the prevalence of comorbidities by age (score test for trend, chi-square: 129.1, p-value: 0.0001). Compared to those $<55$ years of age, CRC patients aged $\geq 75$ years showed a nearly eightfold higher prevalence of congestive heart failure and 
a nearly six-fold higher prevalence of type-II diabetes mellitus and dementia. The prevalence of comorbidities was higher among men than among women. Diabetes and COPD were the most common comorbidities among men. However, women showed a higher prevalence of dementia and rheumatologic disease ( $6.6 \%$ vs $3.3 \%$ and $15.1 \%$ vs $6.7 \%$ ). Among the top five comorbidities plus dementia, congestive heart failure had the highest prevalence among patients with restricted performance status (34.3\%) and disability (66.7\%), whereas COPD had the highest prevalence among current and previous smokers. There was strong evidence supporting a significant trend in the prevalence of comorbidities across the levels of performance status for the five most common comorbidities plus dementia (score test for trend, chi-square: 71.5, p-value: 0.0001) (Table 1).

Furthermore, CRC patients with COPD and diabetes showed the highest prevalence of current smokers $(27.0 \%$ and $23.8 \%$, respectively). Current smokers among CRC patients with COPD were 2.5 more prevalent than neversmokers. CRC patients with diabetes showed the highest prevalence of overweight and obesity $(22.7 \%$ and $31.1 \%$, respectively) while CRC patients with dementia had the lowest prevalence (Table 1).

\section{Tumor Factors}

Overall, the tumor factors showed a weak association with a higher prevalence of comorbidity at diagnosis. The most common anatomical sites for the top five comorbidities plus dementia were the right and left sides of the colon. Compared to the other comorbidities, dementia showed the highest ratio of grade IV vs grade I of differentiation (Table 1).

\section{Comorbidity and Multimorbidity by Patient and Tumor Factors}

Table 2 shows the PRs of multimorbidity and comorbidity vs the absence of comorbidities at diagnosis by patient and tumor factors. Overall, male CRC patients who were older, obese, current smokers, and with restricted performance status or disability had a higher prevalence of multimorbidity. For instance, the prevalence of multimorbidity was 2.7 times higher in current smokers (95\% CI: 1.6-4.8) than that in non-smokers while the prevalence of multimorbidity in obese CRC patients was 2.4 times (95\% CI: 1.4, 4.0) that in CRC patients with a BMI $<25 \mathrm{~km} / \mathrm{m}^{2}$ at diagnosis (Table 2).

\section{Time-to-Surgery}

Figure 1A shows a right-skewed distribution of the time from CRC diagnosis to surgery. The minimum time-tosurgery was 0 days in 171 patients $(11 \%)$, representing a $\mathrm{CRC}$ diagnosis during surgery. The maximum time was 587 days in one patient. Among patients with diagnosis at surgery, $46 \%$ did not have comorbidities, $34 \%$ had one comorbidity, and $20 \%$ had multimorbidity. The 5th, 25th, 50th, 75th, and 95th percentiles of time-to-surgery were 0 , 5, 35 (median), 80, and 177 days, respectively. The observed mean and standard deviation for the time-tosurgery were 55 (95\% CI: 52-61 days) and 66 days, respectively. The median and IQR for time-to-surgery by comorbidity status were: 30 days (IQR: 89 ) for no comorbidities, 31 days (IQR: 65) for one comorbidity, and 46 days (IQR: 69) for multimorbidity (Figure 1B).

Table 3 shows the estimated difference in time-tosurgery for one comorbidity and multimorbidity compared to that in the group with no comorbidity, adjusted for patient age and disease stage. The time-to-surgery for patients with one comorbidity was 5.2 days $(95 \% \mathrm{CI}$ : -1.3-11.6) longer than that for patients without comorbidity; however, this difference was not statistically significant. In contrast, we detected a significantly longer time-to -surgery in patients with multimorbidity than that in patients without comorbidity (16.7 days longer; 95\% CI: 3.2-29.4). Figure 2 shows the predicted marginal mean of time-to-surgery by patient comorbidity status adjusted for age and stage. Overall, multimorbidity increased the time from cancer diagnosis to surgery across all ages and stages (Figure 2).

\section{Discussion}

We identified a particular pattern of patient and tumor factors associated with a higher prevalence of comorbidities and multimorbidity at diagnosis among CRC patients in two Spanish provinces and an increased time-to-surgery in patients with multimorbidity compared to that in those without comorbidities. The prevalence of multimorbidity at diagnosis was higher in male CRC patients with advanced age, restricted performance or disability, obesity, and smoking habits.

There is a scarcity of literature reporting the prevalence of diabetes among CRC patients. ${ }^{27}$ However, we found that diabetes was the most prevalent comorbidity among CRC patients (24\%). Among non-cancer populations, the prevalence of diabetes in adults in Spain ranges between 
Table I Distribution and Frequency of Patient and Tumor Factors for the Top Five Comorbidities Plus Dementia by Patient and Tumor Factors Among Colorectal Cancer Patients at Diagnosis in Granada and Girona, 201 I, n = I06 I

\begin{tabular}{|c|c|c|c|c|c|c|c|c|c|c|c|c|c|}
\hline & \multirow[t]{2}{*}{ Total } & \multicolumn{2}{|c|}{$\begin{array}{l}\text { Congestive } \\
\text { Heart } \\
\text { Failure }\end{array}$} & \multicolumn{2}{|c|}{$\begin{array}{l}\text { Peripheral } \\
\text { Vascular } \\
\text { Disease }\end{array}$} & \multicolumn{2}{|c|}{ Dementia } & \multicolumn{2}{|c|}{ COPD } & \multicolumn{2}{|c|}{$\begin{array}{l}\text { Rheumatic } \\
\text { Disease }\end{array}$} & \multicolumn{2}{|c|}{$\begin{array}{l}\text { Diabetes } \\
\text { Mellitus }\end{array}$} \\
\hline & & $\mathbf{n}$ & $\%$ & $\mathbf{n}$ & $\%$ & $\mathbf{n}$ & $\%$ & $\mathbf{n}$ & $\%$ & $\mathbf{n}$ & $\%$ & $\mathbf{n}$ & $\%$ \\
\hline \multicolumn{14}{|l|}{ Patient's Factors } \\
\hline \multicolumn{14}{|l|}{ Age in Years } \\
\hline$<55$ & 130 & 4 & 3.1 & 9 & 6.9 & 2 & 1.5 & 8 & 6.2 & 6 & 4.6 & 6 & 4.6 \\
\hline $55-64$ & 219 & 17 & 7.8 & 22 & 10.1 & 5 & 2.3 & 21 & 9.7 & 13 & 6.0 & 42 & 19.4 \\
\hline $65-74$ & 272 & 34 & 12.6 & 36 & 13.4 & 5 & 1.9 & 57 & 21.0 & 30 & 11.2 & 86 & 32.0 \\
\hline$\geq 75$ & 440 & 99 & 23.3 & 57 & 13.4 & 36 & 8.5 & 96 & 23.0 & 55 & 12.9 & 116 & 27.3 \\
\hline \multicolumn{14}{|l|}{ Sex } \\
\hline Male & 644 & 96 & 15.2 & 76 & 12.0 & 21 & 3.3 & 143 & 23.0 & 42 & 6.7 & 171 & 27.1 \\
\hline Female & 417 & 58 & 14.1 & 48 & 11.7 & 27 & 6.6 & 39 & 9.5 & 62 & 15.1 & 79 & 19.3 \\
\hline \multicolumn{14}{|l|}{ Performance Status } \\
\hline Normal $(0)$ & 259 & 20 & 7.8 & 12 & 4.7 & 1 & 0.4 & 25 & 9.7 & 19 & 7.4 & 45 & 17.5 \\
\hline Restricted but able to carry out light work (I) & 423 & 68 & 16.1 & 62 & 14.7 & 13 & 3.1 & 89 & 21.0 & 66 & 15.6 & 117 & 27.7 \\
\hline Restricted, unable to work but capable of self-care (2) & 83 & 21 & 25.6 & 10 & 12.2 & 8 & 9.8 & 16 & 20.0 & 9 & 11.0 & 25 & 30.5 \\
\hline Restricted, capable of limited self-care (3) & 35 & 12 & 34.3 & 9 & 25.7 & 6 & 17.1 & 8 & 23.0 & 4 & 11.4 & 14 & 40.0 \\
\hline Disabled (4) & 6 & 4 & 66.7 & 2 & 33.3 & 2 & 33.3 & 2 & 33.0 & 2 & 33.3 & 1 & 16.7 \\
\hline \multicolumn{14}{|l|}{ Smoking Status } \\
\hline Current & 130 & 12 & 9.2 & 15 & 11.5 & 5 & 3.8 & 35 & 27.0 & 9 & 6.9 & 31 & 23.8 \\
\hline Previous & 298 & 55 & 18.5 & 35 & 11.7 & 9 & 3.0 & 80 & 27.0 & 16 & 5.4 & 87 & 29.2 \\
\hline Never & 505 & 68 & 13.5 & 65 & 12.9 & 25 & 5.0 & 54 & 11.0 & 67 & 13.3 & 104 & 20.6 \\
\hline \multicolumn{14}{|l|}{ BMI in $\mathrm{kg} / \mathrm{m}^{2}$} \\
\hline$<25$ & 226 & 17 & 7.5 & 23 & 10.2 & 12 & 5.3 & 40 & 18.0 & 22 & 9.7 & 40 & 17.7 \\
\hline $25.0-29.9$ & 327 & 40 & 12.2 & 42 & 12.8 & 10 & 3.1 & 41 & 13.0 & 25 & 7.6 & 74 & 22.7 \\
\hline$\geq 30$ & 193 & 30 & 15.5 & 33 & 17.1 & 7 & 3.6 & 49 & 25.0 & 21 & 10.9 & 60 & 31.1 \\
\hline \multicolumn{14}{|l|}{ Tumor Factors } \\
\hline \multicolumn{14}{|l|}{ Anatomical Site } \\
\hline Right Colon & 357 & 55 & 15.7 & 44 & 12.5 & 21 & 6.0 & 67 & 19.1 & 31 & 8.8 & 98 & 28.8 \\
\hline Left Colon & 340 & 50 & 14.9 & 41 & 12.2 & 11 & 3.3 & 63 & 18.8 & 33 & 9.9 & 74 & 22.1 \\
\hline Rectal & 353 & 47 & 13.5 & 38 & 10.9 & 16 & 4.6 & 51 & 14.7 & 39 & 11.2 & 76 & 21.8 \\
\hline Colon Unspecified & 11 & 2 & 28.6 & I & 14.3 & 0 & - & 1 & 14.3 & 1 & 14.3 & 2 & 28.6 \\
\hline \multicolumn{14}{|l|}{ Grade } \\
\hline I & 168 & 24 & 15.2 & 19 & 12.0 & 3 & 1.9 & 21 & 13.3 & 19 & 12.0 & 36 & 22.8 \\
\hline ॥ & 596 & 83 & 14.0 & 77 & 13.0 & 27 & 4.6 & 116 & 19.5 & 56 & 9.4 & 138 & 23.3 \\
\hline III & 90 & 13 & 14.6 & 7 & 7.9 & 2 & 2.2 & 12 & 13.5 & 8 & 9.0 & 30 & 33.7 \\
\hline IV & 7 & 1 & 14.3 & 0 & - & 1 & 14.3 & 0 & & 1 & 14.3 & 0 & - \\
\hline \multicolumn{14}{|l|}{ Stage } \\
\hline 1 & 168 & 25 & 15.0 & 18 & 10.8 & 6 & 3.6 & 23 & 13.8 & 17 & 10.2 & 34 & 20.4 \\
\hline II & 281 & 51 & 18.4 & 31 & 11.2 & 13 & 4.7 & 57 & 20.6 & 42 & 15.2 & 69 & 25.0 \\
\hline III & 285 & 29 & 10.4 & 39 & 13.9 & 11 & 3.9 & 54 & 19.3 & 16 & 5.7 & 79 & 28.2 \\
\hline IV & 267 & 43 & 16.2 & 34 & 12.8 & 13 & 4.9 & 37 & 14.0 & 28 & 10.6 & 54 & 20.4 \\
\hline
\end{tabular}

$6 \%$ and $11 \% .^{28}$ Our findings were similar to those previously reported in a Taiwanese cohort of 1197 CRC patients, in which $24 \%$ had a reported history of diabetes. $^{29}$ Diabetes has also been associated with a higher incidence of CRC and a shorter CRC survival. ${ }^{30}$ Therefore, public health programs targeting CRC prevention strategies among diabetic patients might have a positive impact on CRC outcomes in Spain. 
Table 2 Patient and Tumor Factors Associated with Higher Prevalence of Comorbidity (One) and Multimorbidity (Two or More Comorbidities) Among Colorectal Cancer Patients at Diagnosis in Granada and Girona, 20II, $\mathrm{n}=106 \mathrm{I}$

\begin{tabular}{|c|c|c|c|c|c|c|c|}
\hline & \multirow[t]{2}{*}{ Total } & \multicolumn{3}{|c|}{ One Comorbidity vs None } & \multicolumn{3}{|c|}{ Multimorbidity: $\mathbf{2}$ or More vs None } \\
\hline & & $n(\%)$ & $\begin{array}{l}\text { CPR } \\
(95 \% \mathrm{Cl})\end{array}$ & $\begin{array}{l}\text { APR } \\
(95 \% \mathrm{Cl})\end{array}$ & $n(\%)$ & $\begin{array}{l}\text { CPR } \\
(95 \% \mathrm{Cl})\end{array}$ & $\begin{array}{l}\text { APR } \\
(95 \% \mathrm{Cl})\end{array}$ \\
\hline \multicolumn{8}{|l|}{ Patient's Factors } \\
\hline \multicolumn{8}{|l|}{ Age in Years } \\
\hline$<55$ & 130 & $25(19.3)$ & (Reference) & (Reference) & II (8.5) & (Reference) & (Reference) \\
\hline $55-64$ & 216 & $63(29.2)$ & $2.0(1.2,3.4)$ & $2.0(1.2,3.4)$ & $35(16.2)$ & $2.5(1.2,5.3)$ & $2.4(1.2,5.1)$ \\
\hline $65-74$ & 269 & $82(30.5)$ & $3.3(1.9,5.6)$ & $3.2(1.9,5.5)$ & $93(34.6)$ & $8.5(4.3,16.8)$ & $8.2(4.1,16.3)$ \\
\hline$\geq 75$ & 423 & $|3|(3 \mid .0)$ & $4.6(2.8,7.6)$ & $4.6(2.7,7.7)$ & $185(43.7)$ & $14.8(7.6,28.8)$ & $14.9(7.6,29.2)$ \\
\hline \multicolumn{8}{|l|}{ Sex } \\
\hline Male & 630 & $183(29.0)$ & (Reference) & (Reference) & $215(34.1)$ & (Reference) & (Reference) \\
\hline Female & 408 & $118(28.9)$ & $0.8(0.6,1.1)$ & $0.8(0.6,1.1)$ & $109(26.7)$ & $0.6(0.5,0.9)$ & $0.6(0.4,0.8)$ \\
\hline \multicolumn{8}{|l|}{ Performance Status (ECOG) } \\
\hline Normal (0) & 257 & $59(23.0)$ & (Reference) & (Reference) & $40(15.6)$ & (Reference) & (Reference) \\
\hline Restricted but able to carry out light work (I) & 422 & $137(32.5)$ & $2.8(1.9,4.1)$ & $2.5(1.7,3.7)$ & $154(36.5)$ & $4.6(3.1,7.1)$ & $2.0(0.9,4.6)$ \\
\hline Restricted, unable to work but capable of self-care (2) & 82 & $25(30.5)$ & $3.2(1.7,6.1)$ & $2.3(1.1,4.6)$ & $36(43.9)$ & $6.8(3.6,12.8)$ & $7.0(3.3,14.9)$ \\
\hline Restricted, capable of limited self-care or disabled $(3,4)$ & 40 & II (27.5) & $7.4(2.3,24.0)$ & $5.9(1.7,19.6)$ & $25(62.5)$ & $24.7(8.1,75.0)$ & $9.4(4.5,19.7)$ \\
\hline \multicolumn{8}{|l|}{ Smoking Status } \\
\hline Current & 130 & $42(32.3)$ & $1.3(0.8,2.1)$ & $2.1(1.3,3.6)$ & $40(30.1)$ & $1.3(0.8,2.1)$ & $2.7(1.6,4.8)$ \\
\hline Previous & 297 & $89(30.0)$ & $1.4(1.0,2.0)$ & $1.6(1.0,2.5)$ & $113(38.0)$ & $1.9(1.3,2.7)$ & $2.2(1.4,3.4)$ \\
\hline Never & 503 & 145 (28.8) & (Reference) & (Reference) & 137 (27.2) & (Reference) & (Reference) \\
\hline \multicolumn{8}{|l|}{ BMI in $\mathrm{kg} / \mathrm{m}^{2}$} \\
\hline$<25$ & 226 & $72(31.9)$ & (Reference) & (Reference) & $57(25.2)$ & (Reference) & (Reference) \\
\hline $25.0-29.9$ & 326 & $87(26.7)$ & $0.8(0.5,1.2)$ & $0.8(0.5,1.2)$ & $89(27.3)$ & $1.0(0.7,1.5)$ & $1.0(0.6,1.6)$ \\
\hline$\geq 30$ & 193 & $52(26.9)$ & I.I (0.7, I.8) & $1.2(0.7,1.9)$ & $79(40.9)$ & $2.2(1.4,3.5)$ & $2.4(1.4,4.0)$ \\
\hline \multicolumn{8}{|l|}{ Tumor Factors } \\
\hline \multicolumn{8}{|l|}{ Anatomical Site } \\
\hline Right Colon & 357 & $102(29.3)$ & (Reference) & (Reference) & $118(33.9)$ & (Reference) & (Reference) \\
\hline Left colon & 340 & $93(27.8)$ & I.I $(0.7,1.5)$ & $0.9(0.6,1.3)$ & $104(31.0)$ & $1.3(0.9,1.9)$ & $0.8(0.6,1.2)$ \\
\hline Rectal & 353 & $106(30.5)$ & $0.9(0.6,1.3)$ & $1.0(0.7,1.4)$ & $99(28.5)$ & I.I $(0.8,1.6)$ & $0.8(0.5,1.1)$ \\
\hline \multicolumn{8}{|l|}{ Grade } \\
\hline 1 & 158 & $48(30.4)$ & (Reference) & (Reference) & $44(27.8)$ & (Reference) & (Reference) \\
\hline ॥ & 592 & $169(28.6)$ & $1.0(0.6,1.5)$ & $0.9(0.6,1.3)$ & $189(31.9)$ & $1.2(0.8,1.9)$ & $1.0(0.6,1.6)$ \\
\hline III-IV & 96 & $27(28.1)$ & $0.9(0.5,1.6)$ & $0.8(0.4,1.4)$ & $27(28.1)$ & $1.0(0.5,1.8)$ & $0.8(0.4,1.6)$ \\
\hline \multicolumn{8}{|l|}{ Stage } \\
\hline 1 & 167 & $46(27.5)$ & (Reference) & (Reference) & $47(28.1)$ & (Reference) & (Reference) \\
\hline ॥ & 276 & $85(30.1)$ & $1.5(0.9,2.4)$ & $1.3(0.8,2.1)$ & $99(35.9)$ & I.7 (I.0, 2.7) & $1.3(0.8,2.1)$ \\
\hline III & 279 & $76(27.2)$ & I.I $(0.7,1.7)$ & $1.0(0.6,1.7)$ & $89(31.9)$ & $1.2(0.8,1.9)$ & I.I $(0.7,1.8)$ \\
\hline IV & 265 & $85(32.1)$ & $1.3(0.8,2.0)$ & $1.2(0.7,1.9)$ & $72(27.2)$ & $1.0(0.7,1.7)$ & $0.9(0.5,1.5)$ \\
\hline
\end{tabular}

Notes: Age was adjusted for sex and sex was adjusted for age. Complete case analysis: there were 20 (1.9\%) missing values for comorbidities; 256 (24.1\%) for performance status; 128 (12.0\%) for smoking status; 315 (26.7\%) for BMI, and 60 (5.7\%) for stage.

Abbreviations: CPR, crude prevalence ratio; APR, adjusted for age and sex prevalence ratio.

We also observed a high prevalence of stage III/IV. The prevalence was even higher in older CRC patients affected by dementia. These findings may be related to the low utilization of CRC screening in Spain. In 2011, CRC screening programs were implemented in only nine Spanish regions, with partial coverage. ${ }^{32}$ A recent study in Denmark reported a lower prevalence of advanced cancer stage at diagnosis among CRC patients who were offered screening. ${ }^{31}$ However, further research is warranted to explore the status of the implementation of CRC screening in Spain and its public health impact in terms of early diagnosis and cancer stage. While all 
A
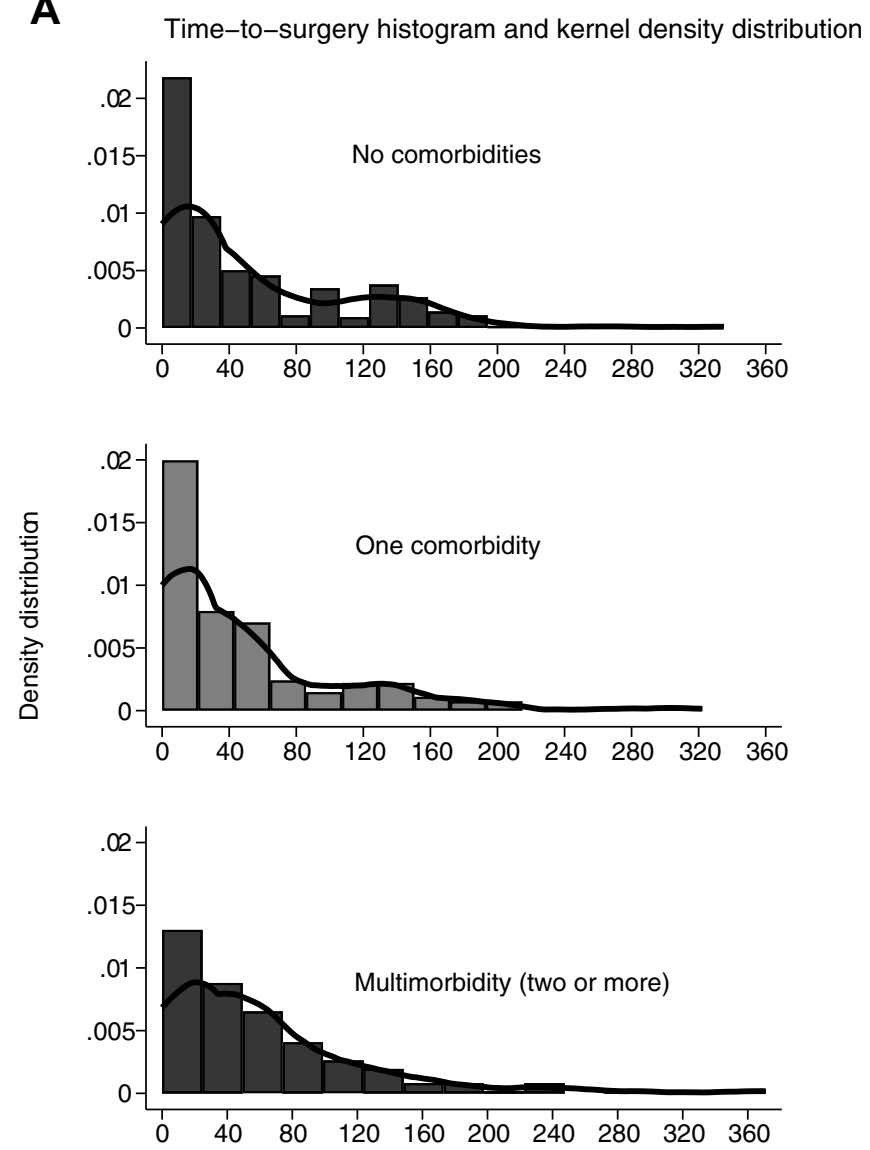

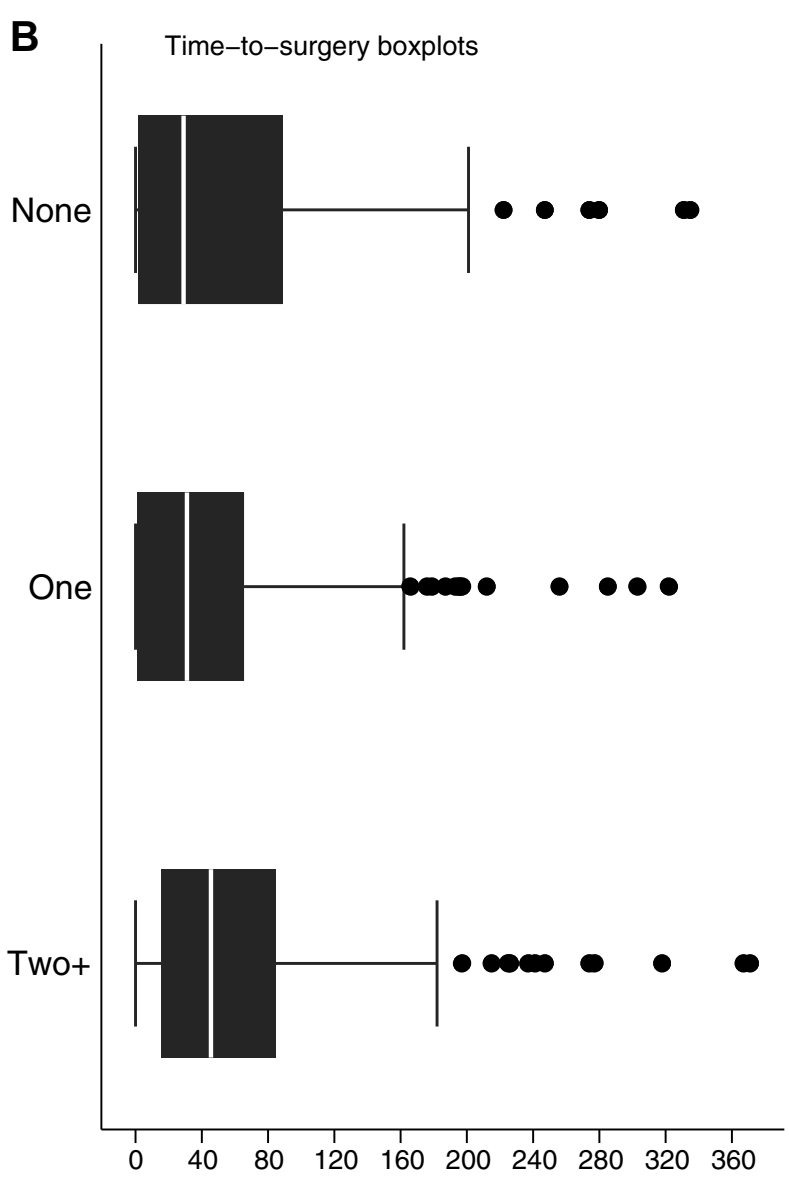

Time from cancer diagnosis to surgery (days)

Figure I Distribution of the time from colorectal cancer diagnosis to surgery by comorbidities status among all incident colorectal cancer patients in Granada and Girona, $201 \mathrm{I}, \mathrm{n}=106 \mathrm{I}(\mathbf{A})$ : Histogram and kernel density and (B): Box plot).

populations would benefit from the systematic use of screening, socioeconomically disadvantaged groups, such as patients with dementia, may especially benefit from targeted CRC screening. ${ }^{33}$

We observed an increasing trend of comorbidities by age. Overall, over $60 \%$ of all cases of cancer are diagnosed after age 65 years of age, with $67 \%$ of cancer deaths occurring in this age group. ${ }^{34}$ Many reasons might explain why cancer occurs more frequently in older persons. The elderly have less resistance and longer exposure to carcinogens, a decline in immunity, altered anti-tumor defenses, decreased DNA repair, defects in tumor-suppressor genes, and differences in biological behavior including angiogenesis. These factors, together with comorbidities, might adversely affect cancer diagnosis, treatment options, and survival in the elderly population. ${ }^{35}$

Given the increased prevalence of multimorbidity in older age groups, health-care professionals need to be vigilant for common comorbidities when offering care for these patients because of the tendency toward poor treatment tolerance and occurrence of complications related to the interaction between age-related physiological changes and comorbidity. ${ }^{35}$ Improved coordination between surgical and medical disciplines is required to optimize the pre-existing comorbid condition for the best cancer survival outcomes and minimal occurrence of treatment complications.

Although cancer stage at the time of CRC diagnosis is a crucial determinant of outcome, comorbidity increases the complexity of cancer management and affects survival. Research on cancer control and treatment should address multimorbidity, particularly in the elderly. ${ }^{36}$ Research is needed to assess the role of comorbidity in the benefitharm ratio of treatment options in elderly patients to improve clinical guidelines to support individualized decision-making in this cohort. 
Table 3 Nonparametric Regression Estimates from Time-toSurgery by Patients' Comorbidity Status, Age and Cancer Stage in Spain 20II, $\mathrm{n}=106 \mathrm{I}$

\begin{tabular}{|c|c|c|}
\hline Variables & $\begin{array}{l}\text { Estimated } \\
\text { CMD }\end{array}$ & $95 \% \mathrm{Cl}^{*}$ \\
\hline \multicolumn{3}{|l|}{ Comorbidities } \\
\hline None & Reference & Reference \\
\hline One & 5.24 & $(-1.31,11.65)$ \\
\hline Two or more & 16.74 & $(3.23,29.42)$ \\
\hline \multicolumn{3}{|l|}{ Age in years } \\
\hline For one-unit increase & $-I . I I$ & $(-1.6,-0.80)$ \\
\hline \multicolumn{3}{|l|}{ Cancer Stage } \\
\hline I & Reference & \\
\hline ॥ & -5.31 & $(-10.68,0.58)$ \\
\hline III & 0.51 & $(-8.43,10.42)$ \\
\hline IV & 1.22 & $(-12.30,17.35)$ \\
\hline
\end{tabular}

Notes: Bold figures highlight statistically significant values (i.e., it does not include the zero). *Bootstrapped $95 \%$ confidence intervals.

Abbreviation: CMD, conditional mean difference from time-to-surgery.

There is no conclusive evidence supporting an optimal window of time from cancer diagnosis to surgical treatment. However, a study from the American College of Surgeons reported that patients who underwent cancer operation at precisely 8 weeks (56 days) after the end of neoadjuvant chemoradiotherapy had the best overall survival and successful removal of their residual tumors. ${ }^{37}$ Another study found that CRC patients waiting for longer than 12 weeks ( 84 days) to receive surgery had an increased all-cause mortality compared to those who received surgery within 4 weeks (28 days). ${ }^{38}$ A study of patients receiving elective surgery for colonic resection following CRC diagnosis in Ontario found that older age and comorbidity were among the factors that influenced the receipt of treatment after 42 days from diagnosis. ${ }^{39}$

The cross-sectional nature of our study design is a limitation and it does not allow for public health recommendations (i.e., targeted CRC screening) or a causal interpretation of the associations given the absence of a temporal link. Another limitation is the absence of information on certain lifestyle characteristics (exercise, diet, and drinking habits) that may also affect the prevalence of lifestyle-related comorbidities. We assumed that data were missing at random and performed a complete case analysis. Only $2 \%$ of data on the main outcome (comorbidities) were missing. Furthermore, the study is based on data from two Spanish provinces, which limits the generalizability of the findings. However, our results are consistent with current evidence regarding the prevalence of comorbidities and, to the best of our knowledge, is the first to identify the most prevalent comorbidities among CRC

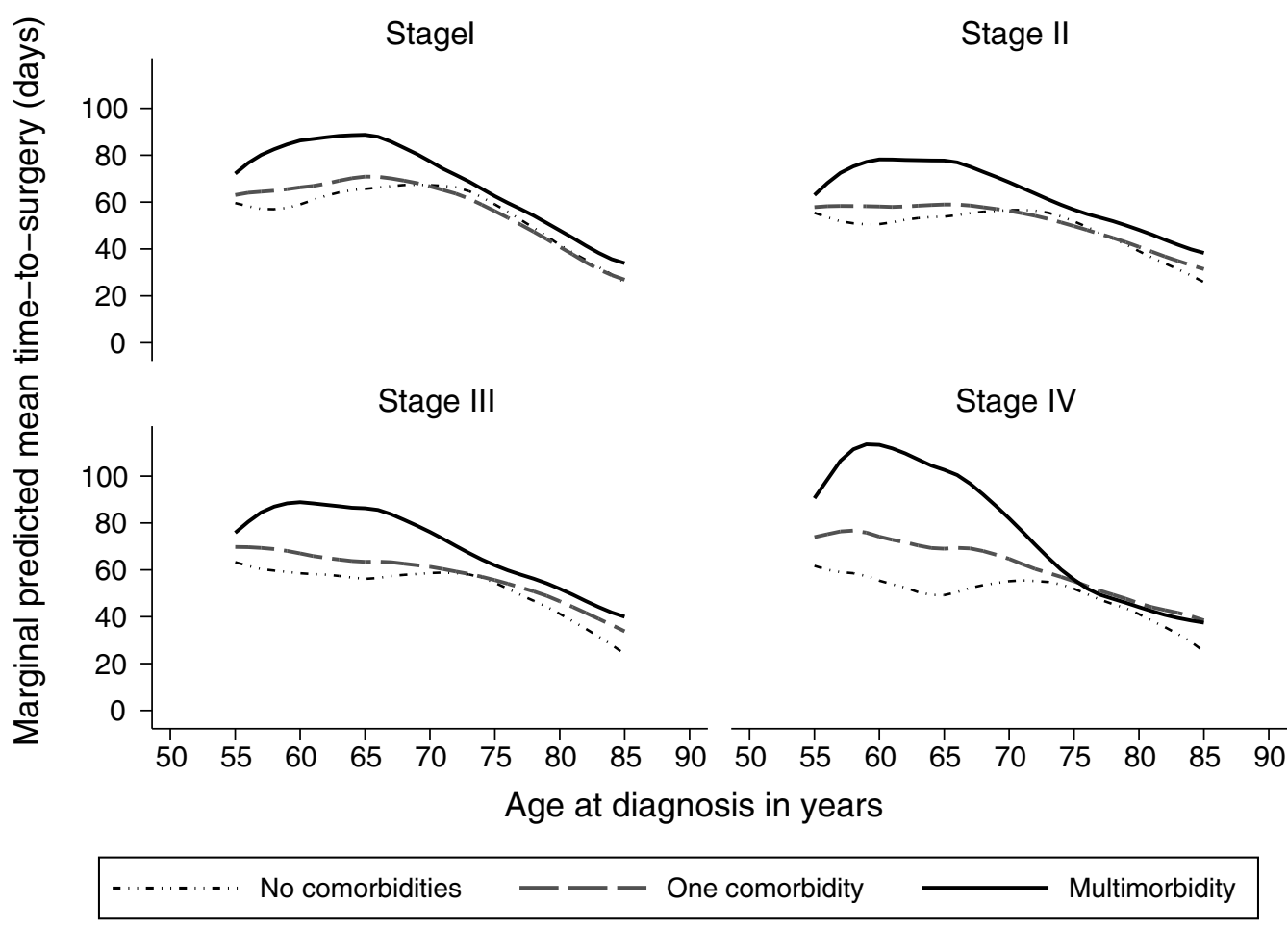

Figure 2 Mean nonparametric estimated time-to-surgery by patients' comorbidity status, age and cancer stage, in Granada and Girona, 20II, $\mathrm{n}=106 \mathrm{I}$. 
patients at diagnosis and to provide evidence of an increased time-to-surgery for CRC patients with multimorbidity in Spain. Furthermore, while clinical studies are representative of only a selected part of the population, ours is a population-based observational study using cancer registry and EHRs data.

Different approaches to measuring comorbidity specifically in cancer patients include focusing on single comorbid conditions in isolation or weighted indices such as the Charlson comorbidity index, ${ }^{40}$ Adult Comorbidity Evaluation - 27 index (ACE-27), ${ }^{41}$ and Elixhauser index. ${ }^{42}$ However, to date, there is no agreed gold standard method upon which to measure comorbidity in the cancer patient population. ${ }^{43}$ We used the Royal College of Surgeons system, which is a clinical score used to evaluate the risk of death during surgery. The score applies equal weights to 12 comorbidities categorized as 0,1 , or 2 or more comorbidities, making it easy to use, since all comorbidities are considered equally important. $^{22}$

In summary, we identified a pattern in the distribution and frequency of patient and tumor factors associated with the higher prevalence of comorbidities and multimorbidity at diagnosis among CRC patients in Spain. Particularly, male CRC patients with advanced age, restricted performance or disability, obesity, and smoking habits had higher prevalence of multimorbidity at diagnosis. Furthermore, CRC patients with multimorbidity showed an increased waiting time from cancer diagnosis to surgery than those without comorbidity. The identification of this pattern may provide insights for further etiological and preventive research and help to identify patients at a higher risk for poorer cancer outcomes and suboptimal treatment.

\section{Acknowledgments}

We thank Drs Minicozzi Pamela and Sant Milena for the development of the protocol and data recollection tools for the European High-Resolution studies.

\section{Funding}

MALF was supported by the Spanish National Health Institute Carlos III (Instituto de Salud Carlos III -ISCIII), Miguel Servet-I Investigator Grant/Award Number: CP17/ 00206-EU-FEDER and MJS for the Andalusian Department of Health, Grant Number: PI-0152/2017. The funders had no role in study design, data collection, and analysis, decision to publish, or preparation of the manuscript.

\section{Disclosure}

The authors have declared that no competing interests exist.

\section{References}

1. Bray F, Ferlay J, Soerjomataram I, Siegel RL, Torre LA, Jemal A. Global cancer statistics 2018: GLOBOCAN estimates of incidence and mortality worldwide for 36 cancers in 185 countries. CA Cancer J Clin. 2018;68(6):394-424. doi:10.3322/caac.v68.6

2. Berkman LF, Kawachi I. Social Epidemiology. Oxford University Press; 2000.

3. Gooden MJ, de Bock GH, Leffers N, Daemen T, Nijman HW. The prognostic influence of tumour-infiltrating lymphocytes in cancer: a systematic review with meta-analysis. Br J Cancer. 2011;105 (1):93-103. doi:10.1038/bjc.2011.189

4. Shenoy P, Harugeri A. Elderly patients' participation in clinical trials. Perspect Clin Res. 2015;6(4):184-189. doi:10.4103/2229-3485.167099

5. Porta MS, Greenland S, Hernán M, Silva I, Last JM, International Epidemiological Association. A Dictionary of Epidemiology. 6th ed. Oxford University Press: Oxford; 2014.

6. Lujic S, Simpson JM, Zwar N, Hosseinzadeh H, Jorm L. Multimorbidity in Australia: comparing estimates derived using administrative data sources and survey data. PLoS One. 2017;12(8): e0183817. doi:10.1371/journal.pone. 0183817

7. Macleod U, Mitchell E. Comorbidity in general practice. Practitioner. 2005;249(1669):282-284.

8. Macleod U, Mitchell E, Black M, Spence G. Comorbidity and socioeconomic deprivation: an observational study of the prevalence of comorbidity in general practice. Eur J Gen Pract. 2004;10(1):24-26. doi:10.3109/13814780409094223

9. Tinetti ME, Fried TR, Boyd CM. Designing health care for the most common chronic condition-multimorbidity. JAMA. 2012;307 (23):2493-2494. doi:10.1001/jama.2012.5265

10. Guthrie B, Payne K, Alderson P, McMurdo ME, Mercer SW. Adapting clinical guidelines to take account of multimorbidity. BMJ. 2012;345:e6341.

11. McLean G, Gunn J, Wyke S, et al. The influence of socioeconomic deprivation on multimorbidity at different ages: a cross-sectional study. Br J Gen Pract. 2014;64(624):e440-e447. doi:10.3399/ bjgp14X680545

12. Sarfati D, Koczwara B, Jackson C. The impact of comorbidity on cancer and its treatment. CA Cancer J Clin. 2016;66(4):337-350. doi: $10.3322 /$ caac.v66.4

13. Gurney J, Sarfati D, Stanley J. The impact of patient comorbidity on cancer stage at diagnosis. Br J Cancer. 2015;113(9):1375-1380. doi:10.1038/bjc.2015.355

14. Stairmand J, Signal L, Sarfati D, et al. Consideration of comorbidity in treatment decision making in multidisciplinary cancer team meetings: a systematic review. Ann Oncol. 2015;26(7):1325-1332. doi:10.1093/ annonc/mdv025

15. Sogaard M, Thomsen RW, Bossen KS, Sorensen HT, Norgaard M. The impact of comorbidity on cancer survival: a review. Clin Epidemiol. 2013;5(Suppl 1):3-29. doi:10.2147/CLEP.S47150

16. Cauley CE, Panizales MT, Reznor G, et al. Outcomes after emergency abdominal surgery in patients with advanced cancer: opportunities to reduce complications and improve palliative care. J Trauma Acute Care Surg. 2015;79(3):399-406. doi:10.1097/TA.0000000000000764

17. Fritz A, Percy C, Jack A, et al. International Classification of Diseases for Oncology. 3rd ed. World Health Organization;2013. Available from:: https://apps.who.int/iris/bitstream/handle/10665/96612/9789241548496_ eng.pdf;jsessionid=776A82AD4DD021FDA6E322900F4E0CEF? sequence $=1$. Accessed January 3, 2020.

18. European High Resolution studies. Available from: http://www.hrstu dies.eu. Accessed January 3, 2020. 
19. Shack L, Jordan C, Thomson CS, Mak V, Moller H. Variation in incidence of breast, lung and cervical cancer and malignant melanoma of skin by socioeconomic group in England. BMC Cancer. 2008;8:271. doi:10.1186/1471-2407-8-271

20. Oken MM, Creech RH, Tormey DC, et al. Toxicity and response criteria of the Eastern Cooperative Oncology Group. Am J Clin Oncol. 1982;5(6):649-655. doi:10.1097/00000421-198212000-00014

21. Maringe C, Fowler H, Rachet B, Luque-Fernandez MA. Reproducibility, reliability and validity of population-based administrative health data for the assessment of cancer non-related comorbidities. PLoS One. 2017;12(3):e0172814. doi:10.1371/journal.pone.0172814

22. Brusselaers N, Lagergren J. The Charlson Comorbidity Index in Registry-based Research. Methods Inf Med. 2017;56(5):401-406. doi:10.3414/ME17-01-0051

23. Edge SB, Compton CC. The American Joint Committee on Cancer: the 7th edition of the AJCC cancer staging manual and the future of TNM. Ann Surg Oncol. 2010;17(6):1471-1474. doi:10.1245/s10434010-0985-4

24. Rothman KJ. Modern Epidemiology. 4th ed. Lippincott Williams \& Wilkins; 2016.

25. Network CRUaNCI. Cancer by deprivation in England: Incidence, 1996-2010, Mortality, 1997-2011. 2014. Accessed 9 May, 2019.

26. Mackenbach JP. Genetics and health inequalities: hypotheses and controversies. J Epidemiol Community Health. 2005;59(4):268-273. doi:10.1136/jech.2004.026807

27. Sarfati D, Gurney J, Lim BT, et al. Identifying important comorbidity among cancer populations using administrative data: prevalence and impact on survival. Asia Pac J Clin Oncol. 2013;12:e47-e56.

28. Shaw JE, Sicree RA, Zimmet PZ. Global estimates of the prevalence of diabetes for 2010 and 2030. Diabetes Res Clin Pract. 2010;87 (1):4-14. doi:10.1016/j.diabres.2009.10.007

29. Huang CW, Sun LC, Shih YL, et al. The impact on clinical outcome of high prevalence of diabetes mellitus in Taiwanese patients with colorectal cancer. World J Surg Oncol. 2012;10:76. doi:10.1186/ 1477-7819-10-76

30. Onitilo AA, Engel JM, Glurich I, Stankowski RV, Williams GM, Doi SA. Diabetes and cancer I: risk, survival, and implications for screening. Cancer Causes Control. 2012;23(6):967-981. doi:10.1007/ s10552-012-9972-3

31. Larsen MB, Njor S, Ingeholm P, Andersen B. Effectiveness of colorectal cancer screening in detecting earlier-stage disease-a nationwide cohort study in Denmark. Gastroenterology. 2018;155(1):99-106. doi:10.1053/j.gastro.2018.03.062
32. Salas Trejo D, Portillo Villares I, Espinas Pinol JA, et al. Implementation of colorectal cancer screening in Spain: main results 2006-2011. Eur J Cancer Prev. 2017;26(1):17-26. doi:10.1097/ CEJ.0000000000000232

33. Mandelblatt J, Andrews H, Kao R, Wallace R, Kerner J. The late-stage diagnosis of colorectal cancer: demographic and socioeconomic factors. Am J Public Health. 1996;86(12):1794-1797. doi:10.2105/ AJPH.86.12.1794

34. White MC, Holman DM, Boehm JE, Peipins LA, Grossman M, Henley SJ. Age and cancer risk: a potentially modifiable relationship. Am J Prev Med. 2014;46(3 Suppl 1):S7-S15. doi:10.1016/j. amepre.2013.10.029

35. Yancik R, Ganz PA, Varricchio CG, Conley B. Perspectives on comorbidity and cancer in older patients: approaches to expand the knowledge base. J Clin Oncol. 2001;19(4):1147-1151. doi:10.1200/ JCO.2001.19.4.1147

36. Yancik R, Wesley MN, Ries LA, et al. Comorbidity and age as predictors of risk for early mortality of male and female colon carcinoma patients: a population-based study. Cancer. 1998;82(11):2123-2134. doi:10.1002/ (SICI)1097-0142(19980601)82:11<2123::AID-CNCR6>3.0.CO;2-W

37. Sun Z, Adam MA, Kim J, Shenoi M, Migaly J, Mantyh CR. Optimal timing to surgery after neoadjuvant chemoradiotherapy for locally advanced rectal cancer. J Am Coll Surg. 2016;222(4):367-374. doi:10.1016/j.jamcollsurg.2015.12.017

38. Shin DW, Cho J, Kim SY, et al. Delay to curative surgery greater than 12 weeks is associated with increased mortality in patients with colorectal and breast cancer but not lung or thyroid cancer. Ann Surg Oncol. 2013;20(8):2468-2476. doi:10.1245/s10434-013-2957-y

39. Flemming JA, Nanji S, Wei X, Webber C, Groome P, Booth CM. Association between the time to surgery and survival among patients with colon cancer: a population-based study. Eur J Surg Oncol. 2017;43(8):1447-1455. doi:10.1016/j.ejso.2017.04.014

40. Charlson ME, Pompei P, Ales KL, MacKenzie CR. A new method of classifying prognostic comorbidity in longitudinal studies: development and validation. J Chronic Dis. 1987;40(5):373-383. doi:10.1016/00219681(87)90171-8

41. Piccirillo JF, Tierney RM, Costas I, Grove L, Spitznagel EL Jr. Prognostic importance of comorbidity in a hospital-based cancer registry. JAMA. 2004;291(20):2441-2447. doi:10.1001/jama.291.20.2441

42. Elixhauser A, Steiner C, Harris DR, Coffey RM. Comorbidity measures for use with administrative data. Med Care. 1998;36(1). doi:10.1097/00005650-199801000-00004

43. Sarfati D. Review of methods used to measure comorbidity in cancer populations: no gold standard exists. J Clin Epidemiol. 2012;65 (9):924-933. doi:10.1016/j.jclinepi.2012.02.017
Clinical Epidemiology

\section{Publish your work in this journal}

Clinical Epidemiology is an international, peer-reviewed, open access, online journal focusing on disease and drug epidemiology, identification of risk factors and screening procedures to develop optimal preventative initiatives and programs. Specific topics include: diagnosis, prognosis, treatment, screening, prevention, risk factor modification,

Submit your manuscript here: https://www.dovepress.com/clinical-epidemiology-journal systematic reviews, risk \& safety of medical interventions, epidemiology \& biostatistical methods, and evaluation of guidelines, translational medicine, health policies \& economic evaluations. The manuscript management system is completely online and includes a very quick and fair peer-review system, which is all easy to use. 